\title{
Cáncer tiroideo: una causa infrecuente de obstrucción de vía aérea superior en niños. Caso clínico
}

\author{
Lisbeth Platzer $M^{1}$, Luis E Vega-Briceño ${ }^{2}$, \\ Hernán G onzález $D^{3}$, Fernando Iñiguez $0^{4 a}$, \\ Cyntia Escobar F5a, Francisco Prado A $^{2}$. \\ Thyroid carcinoma as a cause of \\ upper airway obstruction in children. \\ Case report
}

Upper airway obstruction (UAO) can be a severe medical condition with a high mortality in children. We report a 10 year-old girl with UAO due to papillary thyroid carcinoma. The study confirmed a thyroid cancer. The patient was referred to our centre for the evaluation of dyspnea and hoarseness. She was admitted in severe respiratory distress. Her chest X-ray revealed a critical narrowing of the cervical trachea and extensive infiltration of the lung with a miliary pattern; CT scan revealed a thyroid mass with bilateral pulmonary dissemination. An early surgical approach with total thyroidectomy and tracheotomy was performed. The study revealed a thyroid carcinoma. The patient was then referred to a specialized centre to receive chemotherapy. Recognition of thyroid carcinoma in children requires a high suspicion index. An early CT scan and fiberoptic assessment could show UAO in many unsuspected lesions (Rev Méd Chile 2006; 134: 772-6).

(Key words: Airway obstruction; Thyroid neoplasms; Thyroidectomy)

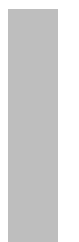

\begin{abstract}
Recibido el 18 de agosto, 2005. Aceptado el 26 de diciembre, 2005.
${ }^{1}$ Consultorio Madre Teresa de Calcuta, Red Salud Familiar, Pontificia Universidad Católica de Chile. ${ }^{2}$ Departamento de Pediatría, Pontificia Universidad Católica de Chile. ${ }^{3}$ Sección de Oncología, División de Cirugía, Pontificia Universidad Católica de Chile. ${ }^{4}$ Programa de Enfermedades Respiratorias del Niño, Pontificia Universidad Católica de Chile. ${ }^{5}$ Departamento de Anatomía Patológica, Pontificia Universidad Católica de Chile. Santiado de Chile. aBecado
\end{abstract}

L a obstrucción de la vía aérea superior (OVAS) constituye una condición médica con un espectro clínico variable desde el ronquido o estridor congénito, hasta la dificultad en mantener una adecuada ventilación espontánea 0 asistida ${ }^{1}$. La

Correspondencia a: Dr. Luis E. Vega-Briceño. Lira 85, 5음 piso, Santiago Centro. Casilla 114-D. Departamento de Pediatría, Pontificia Universidad Católica de Chile. Teléfono: 354-3767. E mail: levega@puc.cl
OVAS crítica representa la dificultad en lograr una intubación endotraqueal, por lo que es una emergencia médica que requiere un rápido reconocimiento por el riesgo de paro cardiorrespiratorio y secuelas neurológicas permanentes ${ }^{2}$. Una evaluación clínica completa representa la aproximación inicial frente a cualquier paciente con OVAS.

Las masas tumorales cervicales y cérvico-torácicas con extensión al mediastino antero-superior pueden desplazar y eventualmente colapsar la vía 
aérea superior (VAS). Si bien el linfoma es la causa más frecuente, existen otras condiciones: linfangioma quístico, tumores vasculares, bocio congénito 0 adquirido, teratomas, tumores del saco vitelino y células germinales ${ }^{3,4}$. Las malformaciones congénitas como quistes broncogénicos son muy raras y se presentan en los lactantes 4 . Otros tumores con adenopatías metastásicas, se observan habitualmente en niños mayores ${ }^{4,5}$.

El cáncer tiroideo es una condición poco frecuente en pediatría, representando 1,5\% de todos los tumores y $7 \%$ de todas las neoplasias de cabeza y cuello ${ }^{5}$. Nuestro objetivo es presentar un caso pediátrico infrecuente de OVAS crítica, secundario a un carcinoma tiroideo, brindando una breve revisión de la literatura en torno al tema.

\section{CASO CĹNICO}

Paciente escolar de 10 años, sin antecedentes mórbidos, consultó con historia crónica de 9 meses de disfagia, disfonía y disnea a esfuerzos progresiva, que le impidió continuidad en el coro de su colegio. El examen físico inicial descrito era normal. Durante 6 meses recibió tratamiento con corticoides inhalados en dosis habituales por baja sospecha de asma, sin mostrar remisión de los síntomas. Posteriormente, apareció una voz más apagada, susurrada, con mayor dificultad respiratoria y ortopnea evidente. La primera radiografía de tórax reveló un patrón de infiltración intersticial miliar de tipo micronodular, bilateral (Figura 1). En la región cérvico-torácica se observó un estrechamiento filiforme de la VAS en forma de lápiz», planteándose como primer diagnóstico la posibilidad de un proceso expansivo vascular o neoplásico.

La noche previa a su traslado, la niña presentó apnea y cianosis durante el sueño, por lo que ingresó a la Unidad de Cuidados Intensivos del Hospital Clínico de la Pontificia Universidad Católica de Chile. Al ingreso, destacó una frecuencia cardiaca: $120 \mathrm{x}^{\prime}$, frecuencia respiratoria: $36 \mathrm{x}^{\prime}$, $\mathrm{SatO}_{2}$ : 88-90\% ( $\mathrm{FiO}_{2}$ ambiental), afebril, con dificultad respiratoria severa, preferentemente inspiratoria, uso de musculatura accesoria cervical y tiraje intenso, que empeoraba con el decúbito supino. A

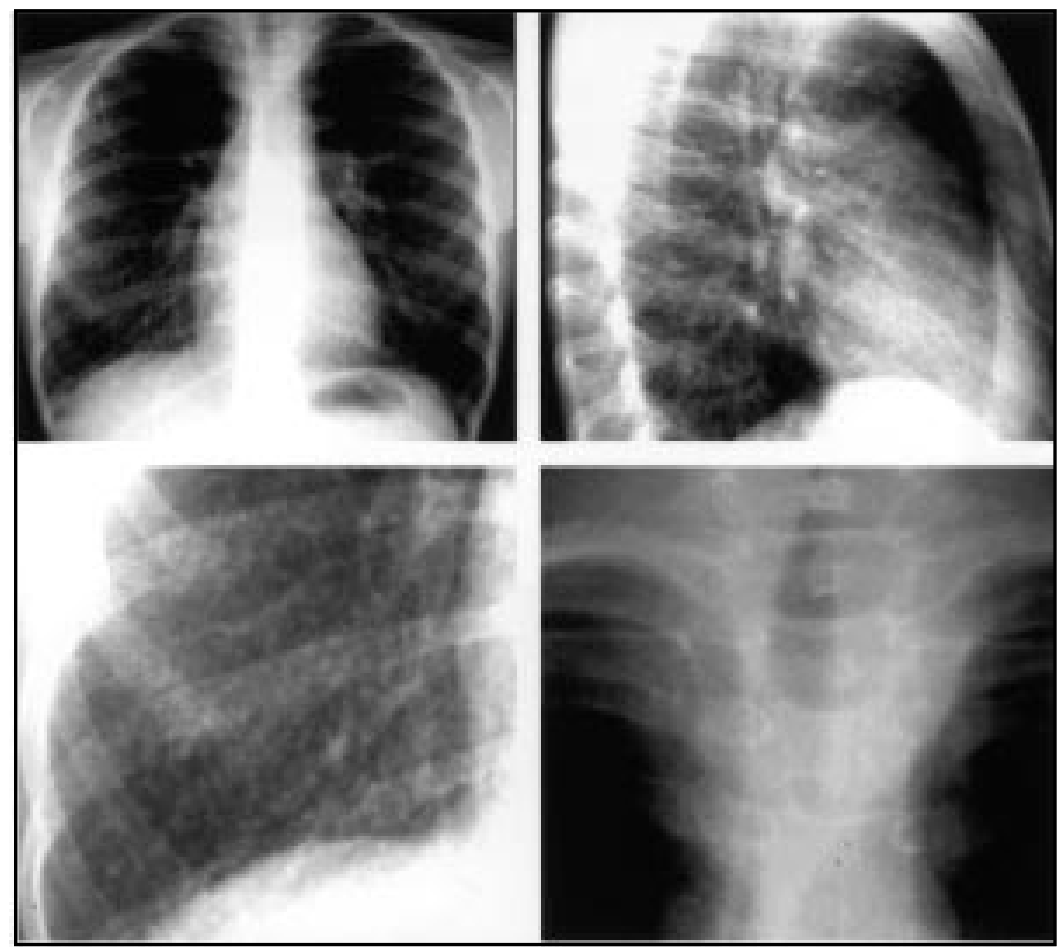

Figura 1. Radiografía de tórax AP y lateral que demuestra la presencia del patrón intersticial de tipo miliar. Nótese la obstrucción de aspecto filiforme a nivel de la vía aérea en «orma de lápiz». 
nivel cervical, se palpó una tiroides de tamaño normal (ausencia de bocio) con consistencia levemente aumentada. El examen pulmonar fue normal, sin ruidos patológicos. Por la gravedad y severa limitación al flujo inspiratorio, se desestimó evaluar la función pulmonar. Se realizó una nueva radiografía cérvico-torácica que confirmó los hallazgos iniciales. No se hizo ecografía, prefiriéndose realizar una tomografía axial computada (TAC) helicoidal, de corte ultrarrápido, sin sedación, la cual reveló la presencia de una extensa masa tumoral en la región cervical anterior y mediastino antero-superior con compresión crítica de la tráquea y medialización de la cuerda vocal derecha, sugerente de una parálisis cordal (Figura 2). Ambos pulmones mostraron imágenes micronodulares sugerentes de metástasis pulmonares.

Dado el alto riesgo de perder la VAS al momento de la inducción anestésica y a la gran ansiedad de la niña, se procedió a una intubación semiconsciente. Se realizó una tiroidectomía total, disección cervical linfática bilateral, resección parcial de pared esofágica, cartílago laríngeo y nervio laríngeo recurrente derecho. Dado el compromiso tumoral de ambos nervios laríngeos recurrentes se realizó una traqueostomía (TQ). La evolución postoperatoria fue satisfactoria, permitiendo su salida de ventilación mecánica luego de $24 \mathrm{~h}$. El estudio anatomopatológico reveló un carcinoma tiroideo papilar bien diferenciado, con patrón predominante folicular (Figura 3), decidiéndose diferir el inicio del tratamiento con radioyodo por 3-4 semanas por la presencia del medio de contraste administrado en la TAC. La niña fue trasladada en buenas condiciones a otro hospital para el tratamiento oncológico definitivo. Posteriormente, en un control endoscópico a las tres semanas, se evidenció la movilidad completa de la cuerda vocal izquierda, lo que permitió decanular a la paciente con recuperación aproximadamente de $60 \%$ del tono de la voz y sin mostrar trastornos de la deglución.

\section{DisCUSIÓN}

Este caso demuestra una causa infrecuente de OVAS crítica en una escolar con disfagia y disfonía crónica asociada a dificultad respiratoria progresiva secundaria a un carcinoma maligno tiroideo. Su reconocimiento requirió un alto índice de sospecha clínica por la presencia de síntomas inespecíficos. En

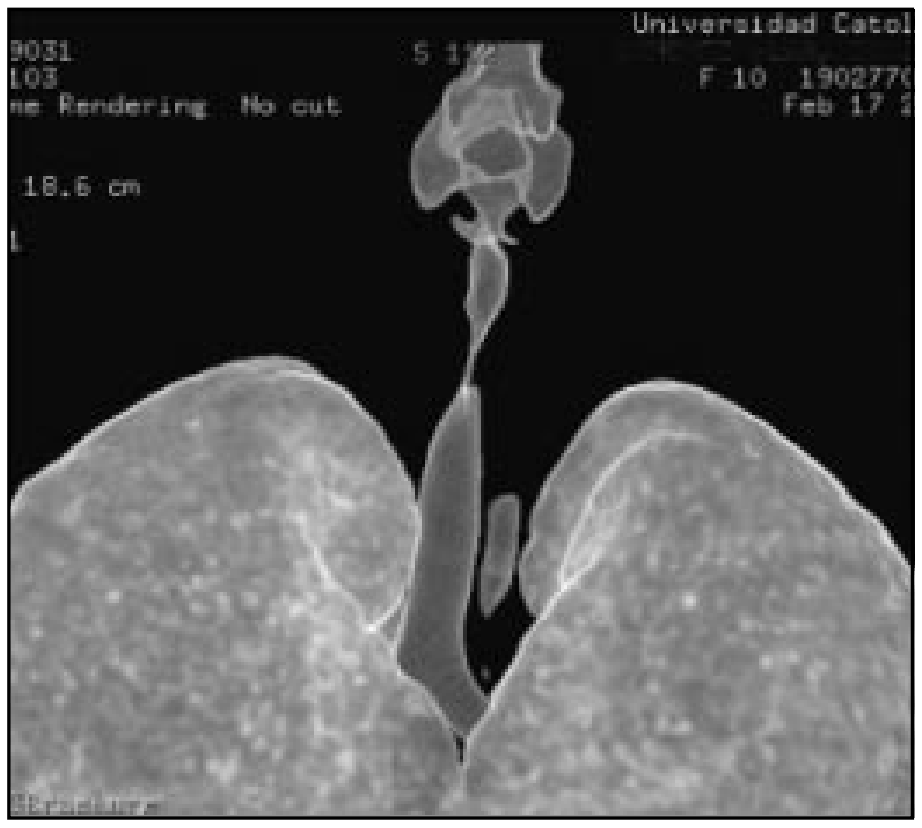

Figura 2. Tomografía helicoidal con reconstrucción de la vía aérea que revela la presencia de una extensa masa tumoral en la región cervical anterior y mediastino antero-superior con compresión crítica de la tráquea. 


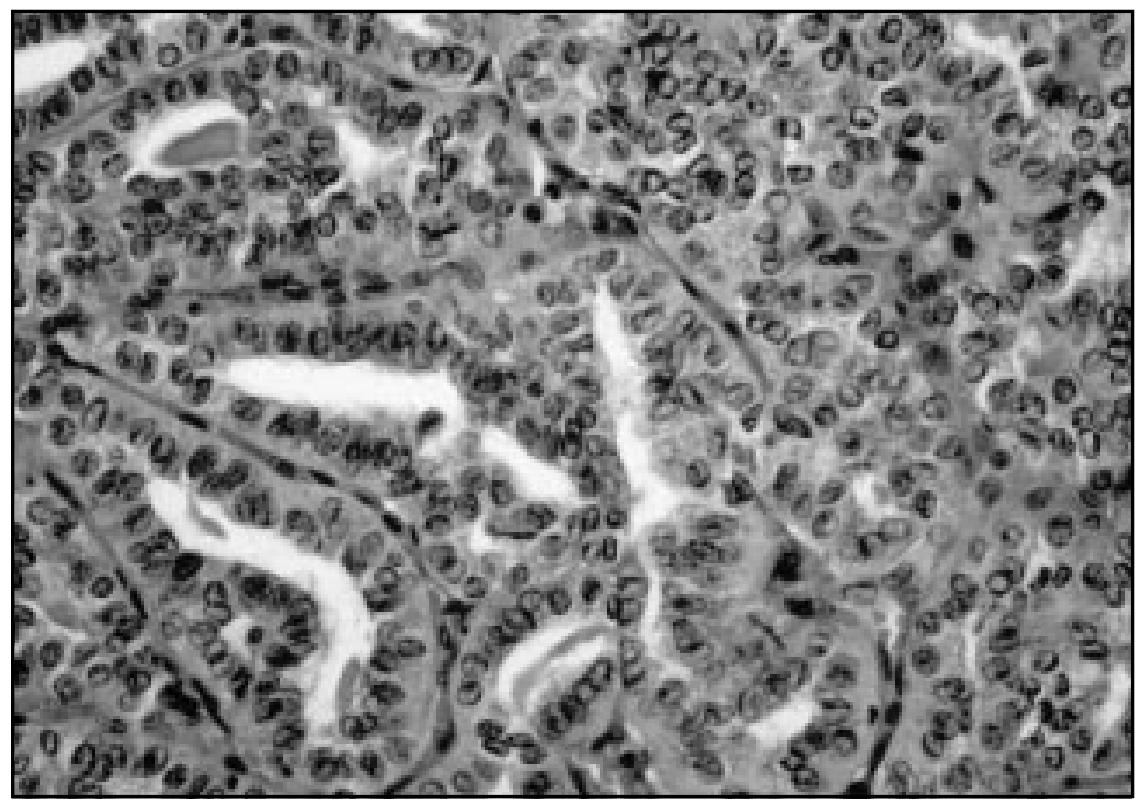

Figura 3. Tinción hematoxilina eosina $(40 \mathrm{x})$ que demuestra la formación de estructuras foliculares, separadas por tabiques fibroconjuntivos, con escaso material coloideo intraluminal, revestidas por células cuboides, de núcleos claros, de contomos imegulares, sobrepuestos, algunos con pliegues 0 escotaduras centrales y pseudoinclusiones.

nuestra paciente, la radiografía de tórax fue útil en precisar imágenes intersticiales de aspecto miliar y demostrar el estrechamiento del aerograma en la región cervical de la tráquea, alertando la posibilidad de un cáncer timoideo con metástasis pulmonares. Creemos que todo niño con OVAS sin una razón evidente, debe ser evaluado de una manera sistemática hasta determinar la causa subyacente.

La presencia de OVAS y un patrón miliar pulmonar en pediatría, sugiere el diagnóstico diferencial de tres condiciones: tuberculosis, linfoma y cáncer tiroideo ${ }^{6}$. El cáncer tiroideo no es una condición excepcional en niños o adolescentes ya que $10 \%$ ocurre en menores de 20 años, presentando hasta $90 \%$ y $33 \%$ de ellos, compromiso ganglionar (preferentemente cervical) y metástasis pulmonares respectivamente ${ }^{5,7}$. A diferencia de los adultos, la preferencia por el sexo femenino es menos clara en niños. La mayoría de pacientes pediátricos se presentan asintomáticos, con una masa firme y móvil en el cuello, sin embargo, la historia de crecimiento rápido sugiere malignidad ${ }^{8}$. Se desconoce la incidencia de cáncer tiroideo como responsable de OVAS en niños o adultos. En general, el carcinoma tiroideo en niños presenta un mejor pronóstico, siendo la edad avanzada y las metástasis pulmonares factores de mal pronóstico ${ }^{7,8}$. La tasa de recurrencia es variable, pudiendo ser hasta $50 \%{ }^{9}$. El tratamiento recomendado es la tiroidectomía total con disección ganglionar selectiva y terapia ablativa con yodo ${ }^{131}$ postoperatoria y supresión de la TSH con levotiroxina ${ }^{5,7}$. La TQ fue un elemento importante para brindar estabilidad de la vía aérea y una apropiada ventilación; esta decisión, consideró el riesgo de compromiso del nervio laríngeo recurrente y la parálisis cordal secundaria a una lesión infiltrativa tumoral o quirúrgica. Paleri y cols reportaron su experiencia con láser como medida paliativa en 14 pacientes adultos con OVAS secundario a cáncer, evitándose el empleo de $\mathrm{TQ}^{10}$.

Una aproximación diagnóstica ordenada y un equipo multidisciplinario en torno a una OVAS crítica, permite optimizar recursos. Actualmente se dispone de herramientas como función pulmonar (curvas flujo volumen, oscilometría de impulso, estudio de ruidos respiratorios); imágenes (fluoroscopia, radiografía cervical anteroposterior y lateral, TAC de alta resolución, programas computacionales con endoscopia virtual, resonancia nuclear magnética [RNM] angiorresonancia) y estudio endoscópico ${ }^{1,2}$. La curva flujo-volumen requiere la colaboración del paciente y permite distinguir si la lesión es extratorácica o intratorácica por el aplanamiento en la forma de la curva en su porción inspiratoria; sin embargo, aunque es muy sensible, la correlación con los síntomas y signos clínicos, radiografía o TAC es limitada ${ }^{11,12}$. La fluoroscopia, 
empleada para estudiar lesiones no identificadas radiográficamente o cuerpos extraños, proporciona una cantidad de radiación considerablemente alta. La introducción de nuevos equipos desplazó su uso, por lo que hoy su empleo es excepcional. La TAC de alta resolución o helicoidal (con reconstitución de imágenes), ha resultado ser útil para evaluar la extensión, compromiso vascular y el grado de estenosis de la vía aérea ${ }^{13}$. A diferencia de la RNM, la TAC no se afecta por el movimiento, brindando una mejor resolución espacial. Su inconveniente radica en la radiación proporcionada y la dificultad en graficar toda la tráquea en su eje axial.

En algunas circunstancias, la endoscopia virtual (EV) podńa ser incluso superior a la fibrobroncoscopia (FBC) en la evaluación de la vía aérea ${ }^{2,11}$, siendo los más beneficiados los pacientes con lesiones fijas de la VAS; sin embargo, no es sensible para evaluar lesiones dinámicas de la vía aérea ${ }^{14}$. Además, debe ser considerada en estenosis graves en donde el FBC no puede ingresar en forma distal, brinda información sobre otras lesiones circundantes a una esteno-

\section{REFERENCIAS}

1. LERNER D, PÉrez-Fontan J. Prevention and treatment of upper airway obstruction in infants and children. Current Opinion Pediatr 1998; 10: 265-70.

2. EBER E. Evaluation of the upper airway. Pediatr Respir Rev 2004; 5: 9-16.

3. Grosfeld JL, Skinner MA, Rescorla FJ, West KW. Mediastinal tumors in children: experience with 196 cases. Ann Surg Oncol 1994; 1: 121-7.

4. Sairahen H, Leigeia M, Louhimo I. Primary mediastinal tumors in children. Eur J Cardiothorac Surg 1987; 1: 148-51.

5. Leboulieux S, Baudin E, Travagl JP, Schlumberger M. Follicular cell derived thyroid cancer in children. Eur J Cancer 2004; 40: 1655-9.

6. Chariot P, Fenz A, Monnet I. Miliary opacities diagnosed as lung metastases of a thyroid carcinoma after 13 years of stability. Chest 1993; S104: 981-2.

7. Danese $D$, Gardin A, Farsetti A, Sciacchitano S, Andreou M, Pontecorvi A. Thyroid carcinoma in children and adolescents. Eur J Pediatr 1997; 156: 190-4.

8. De Keyser LF, Van Herie AJ. Differentiated thymoid cancer in children. Head Neck Surg 1985; 8: 100-14.

9. Grigsby PW, Gal-Or A, Michalski JM, DoherTy GM. Childhood and adolescent thyroid carcinoma. Cancer 2002; 95: 724-9. sis. Si bien la evaluación indirecta de la vía aérea brinda información valiosa, algunas veces se requiere de una evaluación directa endoscópica para determinar la causa específica y guiar una intervención definitiva ${ }^{15}$. El momento de la evaluación endoscópica es variable. La FBC permite tomar muestras de tejidos y secreciones, siendo bien tolerada por el paciente y con una baja tasa de complicaciones ${ }^{16}$. Debe de considerarse siempre la posibilidad de colapso de la VAS por la sedación y el riesgo de una intubación difícil con riesgo de muerte, siendo la máscara laníngea una alternativa ${ }^{17}$.

En resumen, presentamos una causa infrecuente de OVAS crítica secundaria a cáncer tiroideo diseminado, que fue enfrentado en forma secuencial por un equipo multidisciplinario, permitiendo el diagnóstico diferencial en forma rápida y segura. La cirugía es el tratamiento de elección, que requiere una apropiada permeabilidad de la vía aérea en el postoperatorio inmediato y el seguimiento médico para su tratamiento oncológico definitivo.

10. Paieri V, Stafford FW, SAmmut MS. Laser debulking in malignant upper airway obstruction. Head Neck 2005; 27: 296-301.

11. BoISELE PM, ERNST A. Recent advances in central airway imaging. Chest 2002; 121: 1651-60.

12. Melissant C, Smith S, Perdberger R, Verschakelen J, LAMmers J, DemedTs M. Lung function, CT-scan and $\mathrm{X}$-ray in upper airway obstruction due to thyroid goitre. Eur Respir J 1994; 7: 1782-7.

13. Sagy M, Poustchi-Amin M, Nimkoff L, Silver P, SHIKowITZ M, LeONIDAS J. Spiral TC scanning of the chest with three dimensional imaging in the diagnosis and management of paediatric intrathoracic airway obstruction. Thorax 1996; 51: 1005-9.

14. Burke A, Vining D, McGuirt W, Postman G, Browne $\mathrm{J}$. Evaluation of airway obstruction using virtual endoscopy. Laryngoscope 2000; 110: 23-9.

15. Nicolai T. Pediatric bronchoscopy. Pediatr Pulmonol 2001; 31: 150-64.

16. Buic J, Marchac V, Scheinmann P. Complications of flexible bronchoscopy in children: prospective study of 1,328 procedures. Eur Respir J 2002; 20: 1271-6.

17. Naguib M, Streetman D, Cufton S, Nasz S. Use of laryngeal mask airway in flexible bronchoscopy in infants and children. Pediatr Pulmonol 2005; 39: 56-63. 